\title{
DETERMINAÇÃO DE CITRATO DE SILDENAFILA E DE TADALAFILA POR CROMATOGRAFIA LÍQUIDA DE ULTRAEFICIẾNCIA COM DETECÇÃO POR ARRANJO DE DIODOS (CLUE-DAD)
}

\author{
Rafael Scorsatto Ortiz* \\ Superintendência de Polícia Federal no Estado do Rio Grande do Sul, Av. Ipiranga, 1365, 90160-093 Porto Alegre - RS, Brasil \\ Marina Venzon Antunes e Rafael Linden
}

Centro Universitário Feevale, Rodovia RS 239, n. 2755, 93352-000 Novo Hamburgo - RS, Brasil

Recebido em 6/5/09; aceito em 22/7/09; publicado na web em 28/1/10

\begin{abstract}
DETERMINATION OF SILDENAFIL CITRATE AND TADALAFIL BY ULTRA PERFORMANCE LIQUID CHROMATOGRAPHY WITH DIODE-ARRAY DETECTION (UPLC-DAD). A simple ultra-performance liquid chromatographic method for the simultaneous determination of sildenafil citrate and tadalafil was developed and validated. Sample preparation was dissolution in methanol, followed by centrifugation and dilution (1:10) with methanol. Analysis was performed in an Acquity ${ }^{\circledR}$ UPLC system with Acquity ${ }^{\circledast}$ BEH C18 column $(2.1$ x $50 \mathrm{~mm}$, with $1.7 \mu \mathrm{m}$ particles). The elution was isocratic with phosphate buffer $\mathrm{pH}$ 2.3 and acetonitrile $(65: 35, \mathrm{v} / \mathrm{v})$ at a flow rate of $0.7 \mathrm{~mL} / \mathrm{min}$. The method presented adequate specificity, linearity, precision and accuracy and allowed the determination of the drugs in seized forensic samples.
\end{abstract}

Keywords: sildenafil; tadalafil; ultra performance liquid chromatography.

\section{INTRODUÇÃO}

Sildenafila e tadalafila (Figura 1) são fármacos empregados no tratamento da disfunção erétil masculina, por atuarem como inibidores seletivos da fosfodiesterase tipo 5 (PDE-5). ${ }^{1}$ No mercado farmacêutico brasileiro são comercializadas, na forma de comprimidos perorais, as especialidades farmacêuticas Viagra ${ }^{\circledR}$ e Revatio ${ }^{\circledR}$ - contendo a sildenafila na forma do sal citrato, e Cialis ${ }^{\circledR}$ - contendo a tadalafila na forma de base. Segundo a Agência Nacional de Vigilância Sanitária (ANVISA), sobre as especialidades Viagra ${ }^{\circledR}$ e Cialis ${ }^{\circledR}$ atualmente recai a maior incidência de falsificação de medicamentos no país. ${ }^{2}$ Curiosamente, estudos apontam o emprego de inibidores da PDE-5 na adulteração de produtos de origem vegetal de uso lícito, como suplementos alimentares energizantes ou virilizantes, ${ }^{3-5}$ e de uso ilícito, como maconha. ${ }^{6}$
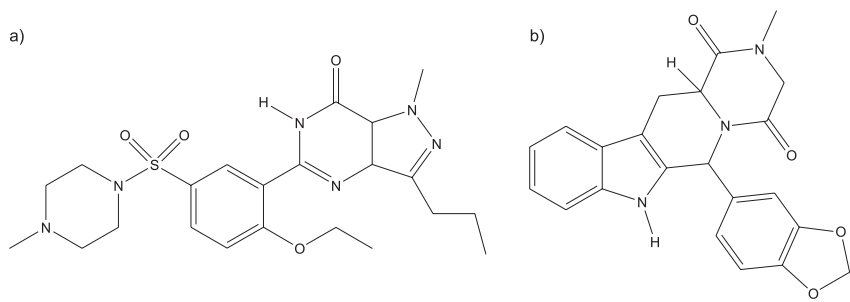

Figura 1. Estruturas químicas da sildenafila (A) e da tadalafila (B)

No âmbito da Criminalística do Departamento de Polícia Federal (DPF), medicamentos contendo o citrato de sildenafila e a tadalafila são objetos de exames periciais que podem ser agrupados, segundo a tipificação penal envolvida, da seguinte forma: falsificação das especialidades farmacêuticas Viagra ${ }^{\circledR}$ e Cialis ${ }^{\circledR}$ e, contrabando/descaminho de medicamentos de origem estrangeira, internalizados de modo irregular no Brasil. Dados do "Sistema Criminalística", que gerencia informações periciais do DPF, mostram que foram emitidos pelo órgão 363 e 104 laudos de exame de produto farmacêutico tendo,

*e-mail: rafaelortiz.rso@dpf.gov.br respectivamente, a sildenafila e a tadalafila como objeto de estudo, no ano de 2008. No período de um ano, foram emitidos pelo Setor Técnico-Científico da Polícia Federal no estado do Rio Grande do Sul (SETEC/SR/DPF/RS) 16 laudos de exame de produto farmacêutico que totalizaram 1570 comprimidos apreendidos (Tabela 1).

A verificação da falsificação de um medicamento fundamenta-se na comparação entre produtos autêntico e questionado, envolvendo uma análise ampla de diferentes elementos, dentre os quais se destacam: a avaliação das embalagens primárias e secundárias; a existência de tinta reativa, que revela um texto ao ser friccionada com objeto metálico; a existência de selos de segurança holográficos; o layout de impressão de bulas e embalagens, incluindo tamanho e tipos de fonte e, a análise externa e interna da forma farmacêutica.

$\mathrm{Na}$ avaliação interna da forma farmacêutica, torna-se essencial a identificação e quantificação do princípio ativo mencionado no rótulo do produto. Deve-se escolher método analítico adequado considerando-se as estabilidades física, química e físico-química das substâncias envolvidas, sob pena de se obterem dados errôneos acerca do produto examinado.

Atualmente não se encontram inseridas em códigos oficiais monografias da sildenafila e da tadalafila. A identificação destas substâncias em formas farmacêuticas autênticas ou falsificadas é realizada com sucesso por diferentes técnicas analíticas, tais como, espectroscopia Raman, espectroscopia de ressonância magnética nuclear $\left(\mathrm{RMN}^{1} \mathrm{H}\right.$, ${ }^{13} \mathrm{C} \mathrm{e}{ }^{15} \mathrm{~N}$ ), difratometria de raios-X, espectrometria no infravermelho próximo e espectrofotometria após complexação. ${ }^{7-12}$

São encontradas inúmeras referências na literatura sobre o emprego da cromatografia líquida de alta eficiência (CLAE) acoplada a diferentes tipos de detectores para determinação quantitativa do citrato de sildenafila e da tadalafila, isoladas ou simultaneamente, a partir de matrizes analíticas de natureza diversa. ${ }^{13-18}$ Em confronto com a cromatografia gasosa, a cromatografia líquida mostra-se vantajosa na execução dos exames periciais solicitados nesses casos por contornar a labilidade térmica do citrato de sildenafila que se decompõe durante a fusão, a $189,4{ }^{\circ} \mathrm{C} \cdot{ }^{5,19,20}$

A cromatografia líquida de ultra eficiência (CLUE) é um dos avanços mais recentes das técnicas de separação; baseia-se nos mesmos princípios da CLAE e utiliza fases estacionárias com partículas 
Tabela 1. Laudos de exame em produtos farmacêuticos contendo citrato de sildenafila ou tadalafila emitidos pelo SETEC/SR/DPF/RS no período de 07/11/2007 a 07/11/2008

\begin{tabular}{|c|c|c|c|c|}
\hline Item & $\begin{array}{l}\text { Medicamento } \\
\text { (Dose) }\end{array}$ & Origem & $\begin{array}{l}\text { Princípio } \\
\text { ativo }\end{array}$ & $\begin{array}{c}\text { Unidades (com- } \\
\text { primidos) }\end{array}$ \\
\hline \multirow[t]{4}{*}{1} & Plenovit (50 mg) & Uruguai & SILD & 20 \\
\hline & Maxfil (50 mg) & Uruguai & SILD & 6 \\
\hline & Vimax (50 mg) & Uruguai & SILD & 100 \\
\hline & Sildenafil Lazar (50 mg) & Uruguai & SILD & 10 \\
\hline 2 & "Viagra" (50 mg) & Brasil & SILD & 132 \\
\hline 3 & Plenovit (50 mg) & Uruguai & SILD & 33 \\
\hline 4 & Pramil (50 mg) & Paraguai & SILD & 9 \\
\hline 5 & Pramil (75 mg) & Paraguai & SILD & 59 \\
\hline \multirow[t]{2}{*}{6} & Plenovit (50 mg) & Uruguai & SILD & 40 \\
\hline & Vimax (50 mg) & Uruguai & SILD & 20 \\
\hline 7 & Pramil (50 mg) & Paraguai & SILD & 10 \\
\hline 8 & Pramil (50 mg) & Paraguai & SILD & 380 \\
\hline \multirow[t]{2}{*}{9} & Pramil Forte (100 mg) & Paraguai & SILD & 84 \\
\hline & Pramil (50 mg) & Paraguai & SILD & 1 \\
\hline \multirow[t]{2}{*}{10} & Libiden (50 mg) & Uruguai & SILD & 404 \\
\hline & Vimax (50 mg) & Uruguai & SILD & 70 \\
\hline 11 & Pramil (50 mg) & Paraguai & SILD & 100 \\
\hline \multirow[t]{2}{*}{12} & "Cialis" (20 mg) & Brasil & TAD & 6 \\
\hline & "Viagra" (50 mg) & Brasil & SILD & 4 \\
\hline \multirow[t]{2}{*}{13} & Pramil (75 mg) & Paraguai & SILD & 5 \\
\hline & "Viagra" (50 mg) & Brasil & SILD & 5 \\
\hline \multirow[t]{2}{*}{14} & Pramil (50 mg) & Paraguai & SILD & 17 \\
\hline & Erofast $(50 \mathrm{mg})$ & Paraguai & SILD & 25 \\
\hline 15 & Pramil $(50 \mathrm{mg})$ & Paraguai & SILD & 20 \\
\hline 16 & Pramil (50 mg) & Paraguai & SILD & 10 \\
\hline Total & & & & 1570 \\
\hline
\end{tabular}

menores que $2 \mu \mathrm{m}$. O uso destas partículas juntamente com as altas velocidades lineares da fase móvel permitem análises muito mais rápidas, na ordem de poucos minutos, consumo de solventes bem menor, eficiências alcançadas mais elevadas, detectabilidade de 2-3 vezes maior, quando comparadas às análises realizadas utilizando-se CLAE. Além disso, a transferência de um método já desenvolvido por CLAE para CLUE pode ser facilmente realizada, deixando de ser um fator limitante para o emprego da CLUE. ${ }^{21}$ Não foram encontradas referências na literatura acerca da aplicação da CLUE à determinação de citrato de sildenafila e/ou tadalafila.

Para garantir que um novo método analítico gere informações confiáveis e interpretáveis sobre a amostra ele deve sofrer uma avaliação denominada validação. ${ }^{22}$ A validação é um processo sistemático que visa assegurar a confiabilidade dos resultados experimentais. Em geral, deve incluir estudos de especificidade, linearidade, exatidão, precisão, sensibilidade, limites de detecção e de quantificação e robustez; além da calibração de equipamentos e de materiais, bem como da qualificação dos analistas. ${ }^{23-25} \mathrm{Em}$ matéria criminal, deve-se acrescer o fato de que dados não fidedignos podem, não somente ser contestados em tribunal, mas também acarretar consequências legais injustas às partes envolvidas. ${ }^{26}$

O presente trabalho descreve um método simples de cromatografia líquida de ultraeficiência com detecção de arranjo de diodos
(CLUE-DAD) para a determinação simultânea de citrato de sildenafila e tadalafila em amostras de comprimidos perorais, com uma preparação de amostra simples e separação isocrática, para identificação e quantificação destes fármacos.

\section{PARTE EXPERIMENTAL}

\section{Materiais}

\section{Padrões de trabalho}

Citrato de sildenafila $(99,9 \%)$ e tadalafila $(99,8 \%)$ foram obtidos, respectivamente, de Laboratórios Pfizer Ltda (São Paulo, Brasil) e Eli Lilly do Brasil Ltda (São Paulo, Brasil) por intermédio do Instituto Nacional de Criminalística (Brasília, Brasil).

\section{Reagentes}

Metanol foi obtido da F. Maia (Cotia, Brasil), acetonitrila grau HPLC e ácido fosfórico 85\% foram obtidos da Merck (Darmstadt, Alemanha) e fosfato de potássio monobásico foi obtido da Nuclear (Diadema, Brasil). Croscarmelose sódica, hipromelose, lactose monoidratada, estearato de magnésio, celulose microcristalina e laurilsulfato de sódio foram obtidos do Laboratório de Desenvolvimento Galênico da Universidade Federal do Rio Grande do Sul (Porto Alegre, Brasil). Água purificada foi obtida através de um sistema Elga Purelab Ultra da Elga Labwater (Lane End, Reino Unido).

\section{Amostras}

Foram avaliados 24 comprimidos encaminhados para exame pericial no Setor Técnico-Científico da Polícia Federal no estado do Rio Grande do Sul. Tais comprimidos foram amostrados a partir de diferentes apreensões ocorridas entre novembro de 2008 e março de 2009, suspeitos de conterem sildenafila ou tadalafila em sua composição. Também foram analisadas amostras comerciais das especialidades farmacêuticas Viagra ${ }^{\circledR}$ e Cialis ${ }^{\circledR}$ adquiridas no mercado farmacêutico local.

\section{Preparação da fase móvel e condições cromatográficas}

A análise foi realizada empregando um sistema de cromatografia líquida de ultraeficiência Acquity ${ }^{\circledR}$, composto de bomba binária, autoamostrador, forno de coluna e detecção por arranjo de fotodiodos e gerenciado pelo programa Empower ${ }^{\circledR}$, todos provenientes da Waters (Milford, EUA).

A fase móvel foi composta de tampão fosfato $10 \mathrm{mM}(\mathrm{pH}$ 2,3) e acetonitrila (70:30, v/v). O tampão fosfato foi preparado através da dissolução de 1,33 g de fosfato de potássio monobásico em $800 \mathrm{~mL}$ de água purificada, seguida da adição de $1 \mathrm{~g}$ de ácido fosfórico $85 \%$ (v/v). O volume foi completado para $1000 \mathrm{~mL}$ com água purificada e o pH foi ajustado com a adição de ácido fosfórico ou hidróxido de potássio $0,1 \mathrm{M}$. Antes da utilização, a fase móvel foi filtrada através de membrana de acetato de celulose com poros de $0,2 \mu \mathrm{m}$ (Waters, EUA) e desgaseificada em banho ultrassônico por 5 min.

A separação foi realizada em uma coluna Acquity ${ }^{\circledR}$ UPLC BEH C18 (2,1 x 50 mm), com diâmetro de partícula de 1,7 micrômetros. A vazão da fase móvel foi $0,7 \mathrm{~mL} / \mathrm{min}$. A coluna foi mantida a 60 ${ }^{\circ} \mathrm{C}$ durante a análise. Foram adquiridos espectros de varredura entre 210 e $400 \mathrm{~nm}$, com monitoramento dos cromatogramas em $290 \mathrm{~nm}$. A corrida cromatográfica teve duração de 1,5 min.

\section{Método}

Solução padrão estoque de citrato de sildenafila e de tadalafila

Cerca de 15,6 mg de citrato de sildenafila e 6,2 mg de tadalafila foram transferidos para balão volumétrico de $25 \mathrm{~mL}$, ao qual foram acrescen- 
tados $22 \mathrm{~mL}$ de metanol. A mistura resultante foi sonicada por $10 \mathrm{~min}$. Após, o volume foi completado com metanol. Foram preparadas duas soluções-estoque: a primeira apresentou a concentração final de 641,76 $\mu \mathrm{g} / \mathrm{mL}$ para citrato de sildenafila e de $254,29 \mu \mathrm{g} / \mathrm{mL}$ para a tadalafila e foi empregada no ensaio de linearidade; e, a segunda, com concentração final de $638,16 \mu \mathrm{g} / \mathrm{mL}$ para citrato de sildenafila e de $260,67 \mu \mathrm{g} / \mathrm{mL}$ para a tadalafila, foi utilizada para os ensaios de precisão e exatidão.

\section{Preparo das amostras}

Após a pesagem individual dos comprimidos em balança analítica, eles foram pulverizados em almofariz de vidro. A partir do pó obtido, uma fração equivalente a $25 \%$ da massa total de cada comprimido foi pesada e transferida para balão volumétrico com capacidade de $25,0 \mathrm{~mL}$, ao qual foram adicionados $22 \mathrm{~mL}$ de metanol. $\mathrm{O}$ balão foi submetido a banho ultrassônico por $5 \mathrm{~min}$, sendo posteriormente completado seu volume com o mesmo solvente. Esta solução intermediária foi transferida para tubo de ensaio e centrifugada a 10.000 g por 5 min à temperatura de $4{ }^{\circ} \mathrm{C}$. Uma alíquota de $1000 \mu \mathrm{L}$ do sobrenadante foi diluída a $10,0 \mathrm{~mL}$ com metanol em balão volumétrico e $5 \mu \mathrm{L}$ foram analisados por CLUE-DAD.

\section{Validação}

Foram avaliados os seguintes parâmetros: especificidade, linearidade, intervalo, precisão (repetibilidade e precisão intermediária) e exatidão.

\section{Especificidade}

Considerou-se o método específico caso não houvesse a presença de picos no mesmo tempo de retenção do citrato de sildenafila (SILD) nem da tadalafila (TAD). Além disto, avaliou-se a pureza dos picos cromatográficos pelos espectros de absorção gerados pela detecção por arranjo de diodos para SILD e TAD.

Preparou-se mistura física contendo $20 \mathrm{mg}$ de cada um dos seguintes adjuvantes farmacêuticos: croscarmelose sódica, hipromelose, lactose monoidratada, estearato de magnésio, celulose microcristalina e laurilsulfato de sódio. Segundo informações das bulas das especialidades farmacêuticas Viagra ${ }^{\circledR}$ e Cialis ${ }^{\circledR}$, tais substâncias integram a composição dos referidos comprimidos. Esta mistura foi transferida para balão volumétrico com capacidade de $25 \mathrm{~mL}$, ao qual foram adicionados $22 \mathrm{~mL}$ de metanol. A seguir, adotou-se processamento idêntico ao descrito em no sub-item Preparo das amostras.

\section{Linearidade e intervalo}

As curvas de calibração foram obtidas através da diluição com metanol da solução padrão estoque de citrato de sildenafila e tadalafila nas concentrações 641,76 para SILD e de $254,29 \mu \mathrm{g} / \mathrm{mL}$ para TAD. Para a construção da curva de calibração, foi utilizada a média de 3 determinações de cada calibrador. Foram utilizados calibradores em 5 níveis diferentes para cada analito. As curvas de calibração foram construídas através do estabelecimento da correlação entre a área dos picos referentes a cada analito (y) e as concentrações adicionadas (x). As curvas foram avaliadas com base nos coeficientes de determinação $\left(\mathrm{R}^{2}\right)$. Foram utilizados calibradores nas concentrações de 38,51 ; 44,$92 ; 51,34 ; 57,76$ e $64,18 \mu \mathrm{g} / \mathrm{mL}$ para SILD e de 15,$25 ; 17,81$; 20,34; 22,89 e 25,43 $\mu \mathrm{g} / \mathrm{mL}$ para TAD, preparados pela diluição de, respectivamente, $1000,900,800,700$ e $600 \mu \mathrm{L}$ de solução padrão estoque dos fármacos a $10 \mathrm{~mL}$ de solução metanólica.

\section{Precisão e exatidão}

A precisão e a exatidão deste método foram avaliadas através de análises em três níveis de concentração para cada analito, realizadas em triplicata e repetidas em 3 dias diferentes. Os calibradores utilizados foram preparados solubilizando-se os padrões de trabalho em metanol. As medidas obtidas para cada nível de concentração foram submetidas à análise de variância (ANOVA) para estimar a precisão intraensaios e a precisão interensaios, ambas expressas como coeficiente de variação porcentual (C.V.\%). ${ }^{26} \mathrm{~A}$ exatidão foi determinada aplicando-se a metodologia analítica proposta na análise dos padrões de referência, a partir dos resultados empregados no cálculo da precisão e foi expressa como percentagem média obtida do valor teórico de cada calibrador. As concentrações utilizadas no estudo de precisão e exatidão foram: 38,29; 51,05 e $63,82 \mu \mathrm{g} / \mathrm{mL}$ para SILD e de 15,$65 ; 20,85$ e $26,07 \mu \mathrm{g} / \mathrm{mL}$ para TAD, preparados pela diluição de, respectivamente, 1000,800 e $600 \mu \mathrm{L}$ de solução padrão estoque dos fármacos $(638,16 \mu \mathrm{g} / \mathrm{mL}$ para SILD e $260,67 \mu \mathrm{g} / \mathrm{mL}$ para TAD) a $10 \mathrm{~mL}$ de solução metanólica.

\section{RESULTADOS E DISCUSSÃO}

A Tabela 1 sumaria os produtos farmacêuticos objeto de exames periciais efetuados pelo SETEC/SR/DPF/RS em período anterior ao início deste trabalho.

No exame de produtos farmacêuticos de origem estrangeira, sem registro ativo no órgão nacional competente (Agência Nacional de Vigilância Sanitária - ANVISA), atualmente entende-se suficiente a caracterização do produto do ponto de vista merceológico. Ou seja, são produtos que não podem ser comercializados no Brasil. $\mathrm{O}$ artigo 12 da Lei Federal $n^{\circ}$ 6.360, de 23 de setembro de 1976, ${ }^{27}$ estabelece que: "nenhum dos produtos de que trata esta Lei, inclusive os importados, poderá ser industrializado, exposto à venda ou entregue ao consumo antes de registrado no Ministério da Saúde."

Entretanto, para produtos com registro ativo para comercialização no Brasil, há a necessidade da avaliação da autenticidade do medicamento. Como são os casos descritos na Tabela 1 como itens 2, 12 e 13, os quais foram objetos de apreensão e de exame produtos denominados "Cialis" e "Viagra", cujos exames periciais realizados à época demonstraram tratar-se de produtos falsificados. Nestes casos, inclusive não é possível afirmar categoricamente que sejam produtos de origem nacional.

Este experimento foi delineado para se obter uma solução intermediária de um comprimido questionado, suspeito de conter sildenafila e/ou tadalafila em doses comerciais convencionais (50 e $20 \mathrm{mg}$, respectivamente), em um volume de $100 \mathrm{~mL}$ de metanol. Na prática, optou-se por reduzir a solução intermediária a $25 \%$ da massa e do volume inicialmente propostos. Posteriormente, uma nova diluição 1:10 com o mesmo solvente fornece a solução de leitura experimental, a qual recai sobre a faixa de linearidade do método, segundo apontaram avaliações preliminares. Ou seja, basta dissolver alíquota do comprimido macerado em metanol, com posterior diluição no mesmo solvente. Trata-se de modo de preparo de amostra simples sendo o usualmente descrito na literatura especializada, cuja única alteração deu-se no emprego do metanol como solvente ao invés da mistura água:acetonitrila (1:1). Tal alteração teve como objetivo diminuir custos, devido à elevação do preço da acetonitrila no mercado de insumos químicos, e não revelou prejuízos à análise.

Acredita-se que o desenvolvimento de um método analítico que permita a determinação simultânea do citrato de sildenafila e da tadalafila tenha utilidade na elucidação de casos forenses nos quais há a substituição de um dos fármacos pelo outro, uma vez que ambos apresentam mesma ação farmacológica. Ou ainda, em exames de comprimido de origem não completamente conhecida, por exemplo, encontrado em uma cena de crime, desprovido de informações completas que caracterizem a especialidade farmacêutica, mas com evidências de se tratar de medicamento que contenha inibidor seletivo da fosfodiesterase tipo 5 (PDE-5). 
A identificação dos analitos foi realizada seguindo-se o difundido por Pragst e colaboradores ${ }^{28}$ para análises cromatográficas por CLAEDAD. Ou seja, associando-se os tempos de retenção à avaliação dos espectros de absorção na região do ultravioleta gerados pelo arranjo de diodos para cada pico (Figura 2) ao passo que a padronização externa foi empregada na quantificação. Devido ao atual estágio de desenvolvimento da tecnologia de arranjo de diodos, os espectros de UV gerados para SILD e para TAD podem ser diferenciados facilmente. ${ }^{3,5,16}$ Isto se credita à alta resolução no comprimento de onda 0,7-1,2 nm/diodo, à exatidão e sensibilidade no comprimento de onda $\lambda \pm 1 \mathrm{~nm}$, à medida de ampla faixa do espectro (de $200 \mathrm{a}$ $400 \mathrm{~nm}$ ) e à comparação computacional com biblioteca de espectros. A identificação de um espectro é feita pela comparação matemática com espectros da biblioteca mediante o cálculo vetorial do índice de similaridade, que inclui todos os pares de dados na faixa de comprimentos de onda medidos (por exemplo, 181 pontos de comparação no caso da faixa de 200 a 380 nm, não somente mínimos e máximos) seguindo um procedimento que independe da concentração. ${ }^{28}$

O espectro do SILD (Figura 2b) apresenta máximos de absorção em torno de 223 e 294, enquanto que o espectro da TAD (Figura 2c) exibe máximos em 220, 283 e 290 nm. Segundo Zou e colaboradores ${ }^{16} \mathrm{o}$ SILD apresenta espectro de UV similar a análogos, tais como, homossildenafila e hidróxissildenafila. Nestes casos, podem ser diferenciados pelos tempos de retenção com subsequente confirmação pelo espectro de massas (EM).

Com o uso da eluição isocrática não foi necessária a etapa de reequilíbrio do sistema antes da nova injeção. Assim, a cromatografia por CLUE, que é intrinsecamente veloz, apresentou tempo de análise consideravelmente curto, com a SILD exibindo retenção em torno de 0,6 min e a TAD com retenção aproximada de 1,2 min. Em termos práticos, quando aplicada à rotina de um laboratório forense, o tempo de análise constitui-se na mais importante vantagem do emprego da CLUE para a análise destes fármacos. Comparado aos métodos descritos na literatura com emprego da CLAE ocorre redução, no mínimo, na ordem de 10 vezes dos tempos de análise, para ambos os fármacos. ${ }^{3,5,13-16,18}$ Tal redução no tempo de análise também acontece em comparação com método de análise por CG, acrescendo-se a vantagem de não exigir derivatização da amostra. ${ }^{29}$

$\mathrm{Na}$ amostra empregada no teste de especificidade, constituída pela mistura física de adjuvantes farmacêuticos (ver sub-item Especificidade) e processada tal qual a amostra questionada, não foram observados picos cromatográficos que não o do metanol (solvente). Além disto, comprovou-se a pureza dos picos cromatográficos das soluções de calibradores e, sobretudo das amostras, avaliada através dos dados espectrais obtidos pela detecção por arranjo de diodos, os quais atribuíram a apenas uma substância cada pico gerado. A conjugação desses fatos permite considerar o método específico, fornecendo alto grau de certeza na identificação dos fármacos, de especial interesse haja vista a aplicação forense do método.

A partir da análise das soluções padrões de concentrações conhecidas de SILD $(38,51$ até $64,18 \mu \mathrm{g} / \mathrm{mL})$ e de TAD $(15,25$ a 25,43 $\mu \mathrm{g} / \mathrm{mL}$ ) obtiveram-se as respectivas áreas dos picos e construíram-se curvas de calibração para cada fármaco. A linearidade foi considerada satisfatória nestas faixas de concentração, uma vez que os coeficientes de determinação $\left(\mathrm{R}^{2}\right)$ foram de 0,9968 para a da $\operatorname{SILD}(\mathrm{y}=12805 \mathrm{x}+$ 9010,9) e de 0,9996 para a da TAD (y = 14066x + 11535).

A precisão intraensaios e a precisão interensaios estiveram entre 0,57 a 2,34\% para SILD e 0,72 a 1,49\% para TAD, demonstrando a elevada precisão do método, especialmente devida ao preparo simplificado de amostra. A exatidão foi determinada em 100\% para SILD e em 99 a $100 \%$ para TAD, também dentro dos critérios de aceitação preconizados pela legislação e normativas internacionais para tais métodos analíticos..$^{21,30} \mathrm{~A}$ Tabela 2 resume os resultados obtidos para precisão e exatidão.
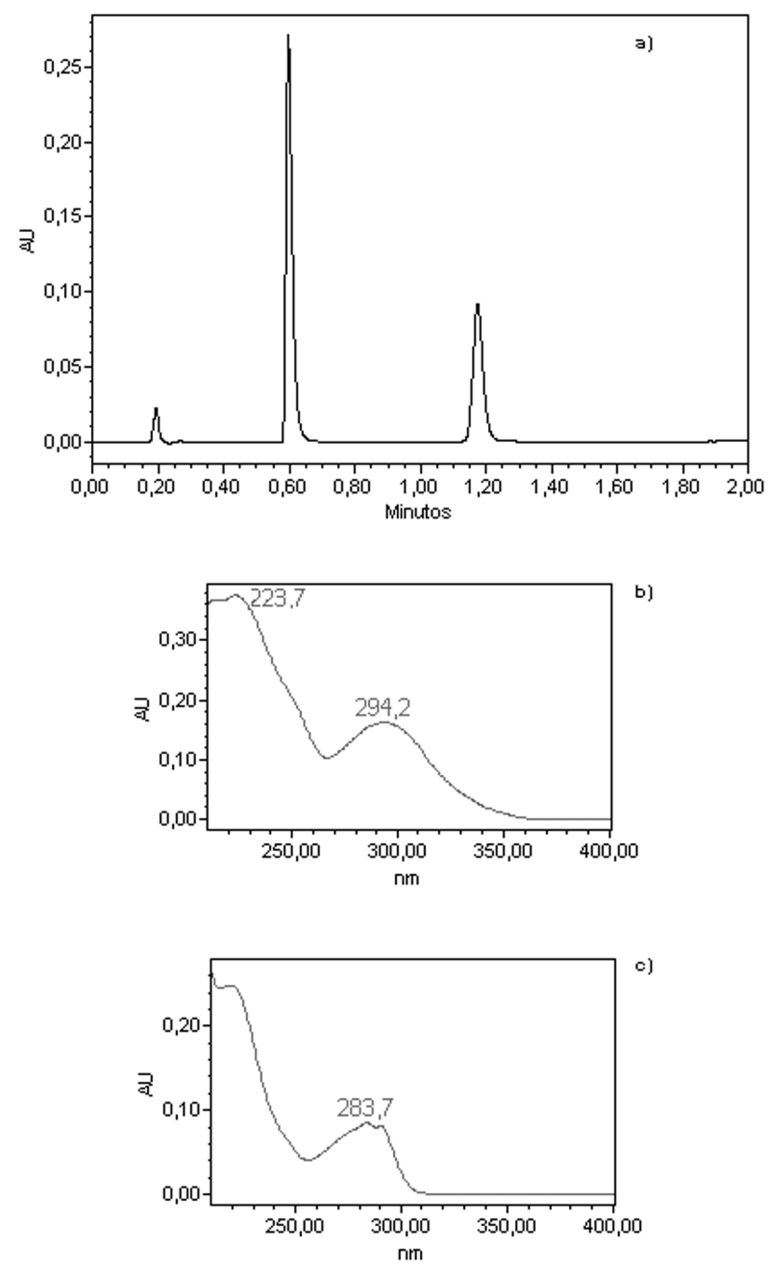

Figura 2. (a) Separação cromatográfica obtida com monitoramento em 290 nm: calibrador $100 \%$ contendo SILD $(51,34 \mu \mathrm{g} / \mathrm{mL})$ e TAD $(20,34 \mu \mathrm{g} / \mathrm{mL})$; (b) espectro UV do pico com retenção em 0,590 min (SILD) e em (c) espectro UV do pico com retenção 1,129 min (TAD) gerados pelo arranjo de diodos

Tabela 2. Resumo do ensaio de precisão e exatidão (três níveis, em triplicata, por três dias)

\begin{tabular}{|c|c|c|c|}
\hline \multicolumn{4}{|c|}{ Citrato de sildenafila } \\
\hline $\begin{array}{l}\text { Concentração } \\
(\mu \mathrm{g} / \mathrm{mL})\end{array}$ & $\begin{array}{c}\text { C.V. intra } \\
\%\end{array}$ & $\begin{array}{c}\text { C.V. inter } \\
\%\end{array}$ & $\begin{array}{c}\text { Exatidão } \\
\%\end{array}$ \\
\hline 38,29 & 0,93 & 1,47 & 100 \\
\hline 51,05 & 0,57 & 2,34 & 100 \\
\hline 63,82 & 0,82 & 1,85 & 100 \\
\hline \multicolumn{4}{|l|}{ Tadalafila } \\
\hline $\begin{array}{l}\text { Concentração } \\
(\mu \mathrm{g} / \mathrm{mL})\end{array}$ & $\begin{array}{c}\text { C.V. intra } \\
\%\end{array}$ & $\begin{array}{c}\text { C.V. inter } \\
\%\end{array}$ & $\begin{array}{c}\text { Exatidão } \\
\%\end{array}$ \\
\hline 15,65 & 0,79 & 1,28 & 100 \\
\hline 20,85 & 0,66 & 1,49 & 99 \\
\hline 26,07 & 0,76 & 0,72 & 100 \\
\hline
\end{tabular}

A Tabela 3 traz os resultados experimentais encontrados para o percentual de fármaco dos 26 comprimidos analisados, considerada a dose teórica rotulada. As amostras 1 até 22 são medicamentos de origem estrangeira, sem registro ativo no Brasil, contendo SILD; as amostras 23 e 24 consistem em falsificações da especialidade farmacêutica "Cialis", nas quais se identificou a TAD; as amostras 
Tabela 3. Percentual de fármaco encontrado nas amostras analisadas

\begin{tabular}{cccccc}
\hline Amostra & $\begin{array}{c}\text { Dose } \\
\text { teórica } \\
(\mathrm{mg})\end{array}$ & $\begin{array}{c}\text { Percentual } \\
\text { de fármaco }\end{array}$ & Amostra & $\begin{array}{c}\text { Dose } \\
\text { teórica } \\
(\mathrm{mg})\end{array}$ & $\begin{array}{c}\text { Percentual } \\
\text { de fármaco } \\
(\%)\end{array}$ \\
\hline 1 & 75 & 97,81 & 14 & 50 & 96,05 \\
2 & 50 & 99,02 & 15 & 50 & 98,65 \\
3 & 50 & 100,41 & 16 & 50 & 59,96 \\
4 & 50 & 97,31 & 17 & 50 & 58,52 \\
5 & 50 & 91,04 & 18 & 75 & 88,69 \\
6 & 50 & 105,90 & 19 & 50 & 71,66 \\
7 & 50 & 89,67 & 20 & 50 & 62,66 \\
8 & 50 & 94,57 & 21 & 50 & 101,74 \\
9 & 50 & 92,58 & 22 & 50 & 95,42 \\
10 & 50 & 98,81 & 23 & 20 & 106,65 \\
11 & 50 & 74,59 & 24 & 20 & 93,69 \\
12 & 50 & 86,73 & 25 & 20 & 98,14 \\
13 & 50 & 68,10 & 26 & 50 & 104,26 \\
\hline
\end{tabular}

25 e 26 são, respectivamente, as amostras comerciais de Cialis ${ }^{\circledR}$ e de Viagra $^{\circledR}$, adquiridas no comércio local.

Das amostras examinadas, 9 apresentaram percentual de fármaco fora da faixa que vai de 90 a $110 \%$ de percentual de fármaco, na verdade abaixo de $90 \%$, chegando-se a encontrar até mesmo valores abaixo de $60 \%$. As falsificações de "Cialis" exibiram entre si uma significativa diferença no percentual de tadalafila, o que já era esperado para produtos de manufatura clandestina. Tais resultados muito provavelmente devem estar associados à ausência ou ineficiência do controle de qualidade na produção dos comprimidos, sendo que processos farmacotécnicos simples, como a adição e homogeneização do princípio ativo com os excipientes, foram pouco eficientes. ${ }^{31}$

\section{CONCLUSÃO}

Um método para a determinação simultânea de citrato de sildenafila e de tadalafila por CLUE-DAD foi desenvolvido e validado. Trata-se do primeiro trabalho relatado com a aplicação de CLUE à determinação destes fármacos. A preparação das amostras foi simples e rápida. $\mathrm{O}$ método foi rápido, específico, linear, preciso e exato na determinação destas substâncias a partir de comprimidos perorais em doses usuais. Do ponto de vista forense trata-se de importante ferramenta com aplicação direta na rotina de exames periciais relacionados ao combate da falsificação e do contrabando/ descaminho de medicamentos.

$\mathrm{O}$ método foi aplicado a amostras de medicamentos de origem estrangeira, irregularmente internalizadas no país, e falsificações de medicamentos encaminhadas à perícia. Detectaram-se importantes variações nos percentuais de fármaco encontrados nos comprimidos examinados. Além disto, algumas amostras apresentaram percentuais de fármaco muito abaixo do rotulado, incluindo percentuais abaixo de $90 \%$ e, até mesmo, valores abaixo de $60 \%$. Ambos os fatos são associados comumente a comprimidos produzidos com qualidade inadequada, inferior à exigida pela legislação brasileira vigente.

\section{AGRADECIMENTOS}

À SENASP/MJ pelo aporte financeiro ao curso de Pós-graduação em Toxicologia Forense - Feevale no qual se insere este trabalho. Ao DPF/MJ pelo incentivo à capacitação profissional. Ao INC/DPF e ao LDG/UFRGS pela doação de reagentes empregados neste trabalho.

\section{REFERÊNCIAS}

1. Hardman, J. G.; Limbird, L. E.; Gilman, A. G.; Goodman e Gilman: As Bases Farmacológicas da Terapêutica, 10ª ed., McGraw Hill: Rio de Janeiro, 2003.

2. http://www.anvisa.gov.br/medicamentos/falsificados/index.htm, acessada em Dezembro 2009.

3. Zou, P.; Oh, S. S.; Hou, P.; Low, M.; Koh, H.; J. Chromatogr., A 2006, 1104,113

4. Reepmeyer, J. C.; Woodruff, J. T.; J. Pharm. Biomed. Anal. 2007, 44, 887.

5. Singh, S.; Gohill, V. M.; Kaur, A.; Prasad, B.; Savalya, A.; Shah, R. P.; Trends Anal. Chem. 2009, 28, 13.

6. Venhuis, B. J.; De Kaste, D.; Forensic Sci. Int. 2008, 182, 23.

7. Dinesh, N. D.; Nagaraja, P.; Made Gowa, N. M.; Rangappa, K. S.; Talanta 2002, 57, 757.

8. Wawer, I.; Pisklak, M.; Chilmonczyk, Z.; J. Pharm. Biomed. Anal. 2005, 38,865 .

9. Vredenbregt, M. J.; Blok-Tip, L.; Hoogerbrugge, R.; Barends, D. M.; De Kaste, D.; J. Pharm. Biomed. Anal. 2006, 40, 840.

10. Maurin, J. K.; Pluci ski, F.; Mazurek, A. P.; Fijałek, Z.; J. Pharm. Biomed. Anal. 2007, 43, 1514.

11. Veij, M.; Deneckere, A.; Vandenabeele, P.; De Kaste, K.; Moens, L.; J. Pharm. Biomed. Anal. 2008, 46, 303.

12. Trefi, S.; Routaboul, C.; Hamieh, S.; Gilard, V.; Malet-Martino, M.; Martino, R.; J. Pharm. Biomed. Anal. 2008, 47, 103.

13. Daraghmeh, N.; Al-Omari, M.; Badwan, A. A.; Jaber, A. M. Y.; J. Pharm. Biomed. Anal. 2001, 25, 483.

14. Dinesh, N. D.; Vishukumar, B. K.; Nagaraja, P.; Made Gowa, N. M.; Rangappa, K. S.; J. Pharm. Biomed. Anal. 2002, 29, 743.

15. Aboul-Enein, H. A.; Ali, I.; Talanta 2005, 65, 276.

16. Cheng, C.; Chou, C.; J. Chromatogr., B 2005, 822, 278.

17. Zhu, X.; Xiao, S.; Chen, B.; Zhang, F.; Yao, S.; Wan, Z.; Yang, D.; Han, H.; J. Chromatogr., A 2005, 1066, 89.

18. Shakya, A. K.; Abuawwad, A. N. A.; Arafat, T. A.; Melhim, M.; J. Chromatogr., B 2007, 852, 403.

19. Melnikov, P.; Corbi, P. P.; Cuin, A.; Cavicchioli, M.; Guimarães, W. R.; J. Pharm. Sci. 2003, 92, 2140.

20. Moffat, A. C.; Osselton, M. D.; Widdop, B.; Clarke's Analysis of Drug and Poisons In Pharmaceutical, Body Fluids and Post Mortem Material, $3^{\text {rd }}$ ed., Pharmaceutical Press: Londres, Estados Unidos, 2004.

21. Maldaner, L.; Jardim, I. C. S. F.; Quim. Nova 2009, 32, 214.

22. Ribani, M; Bottoli, C. B. G.; Collins, C. H.; Jardim, I. C. S. F; Melo, L. F. C.; Quim. Nova 2004, 27, 771.

23. http://www.anvisa.gov.br/legis/resol/2003/re/899_03re.htm, acessada em Dezembro 2009.

24. Mendez, A. S. L.; Dissertação de Mestrado, Universidade Federal do Rio Grande do Sul, Brasil, 2003.

25. Chandran, S.; Singh, R. S. P.; Pharmazie 2007, 62, 4.

26. Peters, F. T.; Drummer, O. H.; Musshoff, F.; Forensic Sci. Int. 2007, 165, 216.

27. http://www.anvisa.gov.br/legis/leis/6360_76.htm, acessada em Dezembro 2009.

28. Pragst, F.; Hezler, M.; Erxleben, B. T.; Clin. Chem. Lab. Med. 2004, 42, 1325.

29. Saisho, K.; Scott, K. S.; Morimoto, S.; Nakahara, Y.; Biol. Pharm. Bull. 2001, 24, 1384.

30. Shah, V. P.; Midha, K. K.; Findlay, J. W.; Hill, H. M.; Hulse, J. D.; McGilveray, I. J.; McKay, G.; Miller, K. J.; Patnaik, R. N.; Powell, M. L.; Tonelli, A; Viswanathan, C. T.; Yacobi, A.; Pharm. Res. 2000, 17, 1551.

31. Da Costa, J. L.; Pintao, E. R.; Corrigliano, C. M. C.; Negrini Neto, O. ; Quim. Nova 2009, 32, 965. 